\title{
Contribution from Star-Forming Galaxies to the Cosmic Gamma-Ray Background Radiation
}

\author{
Makiya Ryu \\ Kyoto University \\ E-mail: makiya@kusastro.kyoto-u.ac.jp \\ Totani Tomonori \\ Kyoto University \\ E-mail: totani@kusastro.kyoto-u.ac.jp
}

\section{Kobayashi A. R. Masakazu}

National Astronomical Observatory of Japan

E-mail: mark.kobayashi@nao.ac.jp

The origin of extragalactic gamma-ray background (EGRB) has been discussed for a long time and various sources have been discussed as possible contributors to EGRB, such as active galactic nuclei, star-forming galaxies, galaxy clusters, structure formation, and dark matter annihilation. We present a new theoretical calculation of the contribution to the EGRB from star-forming galaxies based on a state-of-the-art model of hierarchical galaxy formation that is in quantitative agreement with a variety of observations of local galaxies as well as high redshifts. Gammaray luminosity and spectrum of galaxies are related to star formation rate, gas mass, and star formation mode (quiescent or starburst) of model galaxies using latest observed data of nearby galaxies. Our standard model predicts $\sim 14 \%$ contribution from star-forming galaxies to the total EGRB flux recently reported by the Fermi Gamma-Ray Space Telescope. The predicted number of nearby galaxies detectable by Fermi is consistent with the observation. The sum of the known contributions from AGNs and star-forming galaxies can explain a large part of EGRB, with a remarkable agreement between the predicted model spectrum and observation.

25th Texas Symposium on Relativistic Astrophysics

December 6-10, 2010

Heidelberg, Germany 


\section{Introduction}

Extragalacitc gamma-ray background was first observed by SAS-2 satellite (EGRB[1][2]). The origin of EGRB has been thought that superposition of faint discrete sources, such as active galactic nuclei (AGNs), galaxy clusters, structure formation, and dark matter annihilation. In this work, we calculated the contribution from star-forming galaxies to the EGRB, using the state-of-art model of hierarchical galaxy formation and latest observational data of nearby galaxies. Cosmicrays accelerated by supernova remnants (SNR) are interacted with interstellar gas and radiation field, and emit $\gamma$-rays via bremsstrahlung, inverse Compton (IC), and $\pi_{0}$ decay process. Diffuse gamma-rays from our galaxy and large magellanic cloud (LMC) has been observed, and recently, small magellanic cloud (SMC), M82 and NGC253 are detected in $\gamma$-rays for the first time by H.E.S.S. [3], VERITAS [4], and Fermi [5].

\section{Model}

To calculate the contribution from star-forming galaxies to the EGRB, we need a $\gamma$-ray luminosity function (LF) of galaxies (comoving number density of galaxies per unit $\gamma$ - ray luminosity). We use a mock galaxy catalog produced by one of the latest semi- analytic models of hierarchical galaxy formation (the Mitaka model [6][7]) to construct the LF. The Mitaka model can quantitatively reproduce a wide variety of observed characteristics of galaxies.

We estimated the $\gamma$-ray luminosity of galaxies using star formation rate (SFR) and gas mass. SFR is an indicator of the cosmic-ray luminosity, while gas mass account for the amount of target atom. In Fig 1 it is clearly seen that there is a good correlation between the $\gamma$-ray luminosity and SFR $\times$ gas mass. We fitted these data to power-law function, and the result is

$$
L_{\gamma}=0.28 \times\left(\frac{\mathrm{SFR}}{M_{\odot} \mathrm{yr}^{-1}} \times \frac{M_{\mathrm{gas}}}{10^{9} M_{\odot}}\right)^{0.86}\left[10^{39} \mathrm{erg} / \mathrm{s}\right]
$$

where $L_{\gamma}$ is $\gamma$-ray luminosity in $100 \mathrm{MeV}-5 \mathrm{GeV}$. For the $\gamma$-ray spectrum of galaxies, we simply assumed that all quiescent galaxies have the same spectral shape as the MW. For starburst galaxies, spectrum could become harder than quiescent galaxies, therefore we consider another model that MW-like spectrum plus hard power-law component (see fig 2).

Using the mock numerical galaxy catalog including the SFR and gas mass of each galaxies and eq. (1), we constructed the $\gamma$-ray LF of galaxies. The EGRB flux and spectrum can be calculated by integrating the $\gamma$-ray LF. We also calculated the attenuation of $\gamma$-rays by cosmic infrared background (CIB) photons, and reprocessed cascade emission.

\section{Results}

In Fig 3 the EGRB flux from all star-forming galaxies (quiescent+starburst), as well as the total flux including AGNs, are plotted. Model 1 and 2 correspond to two cases of spectrum of starburst galaxies. For the model of the AGN contribution we used Inoue \& Totani (2009)[8]. The data points are the Fermi measurements of the EGRB including the contribution from resolved sources [9]. To show the effect of absorption, Model 1 with no- absorption and the flux of cascade emission are also 


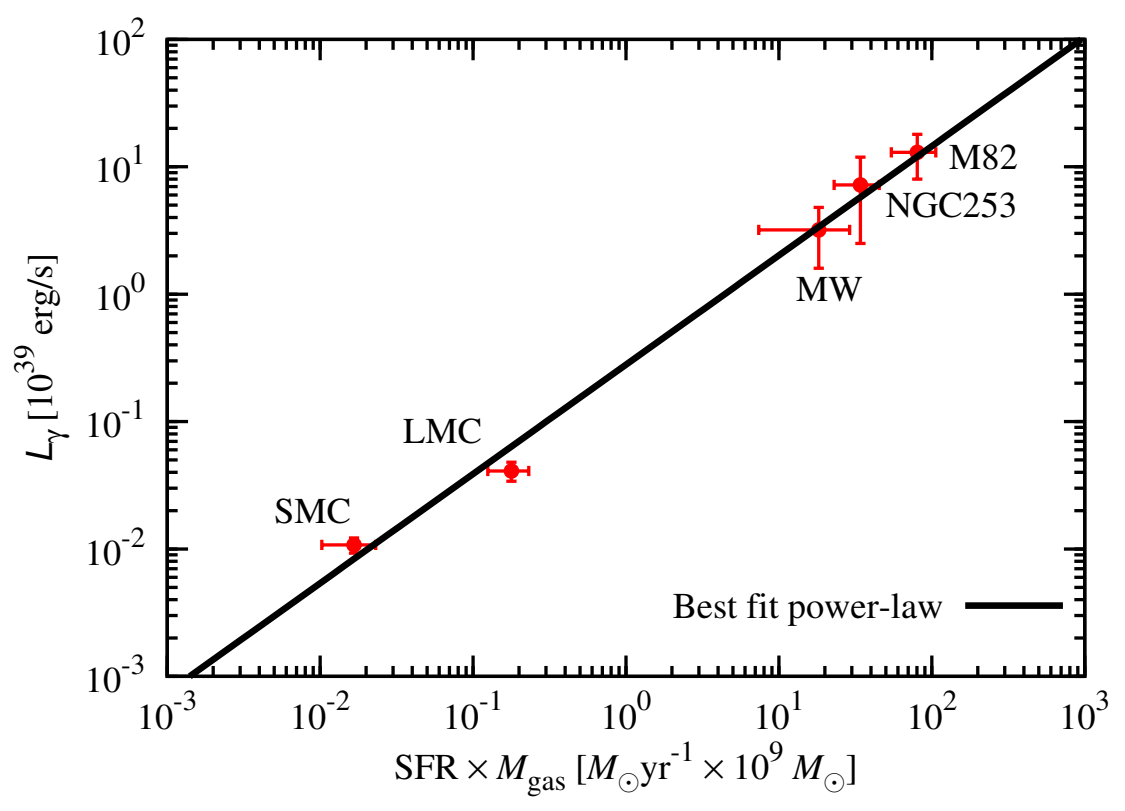

Figure 1: SFR $\times$ gas mass against the $\gamma$-ray luminosity.

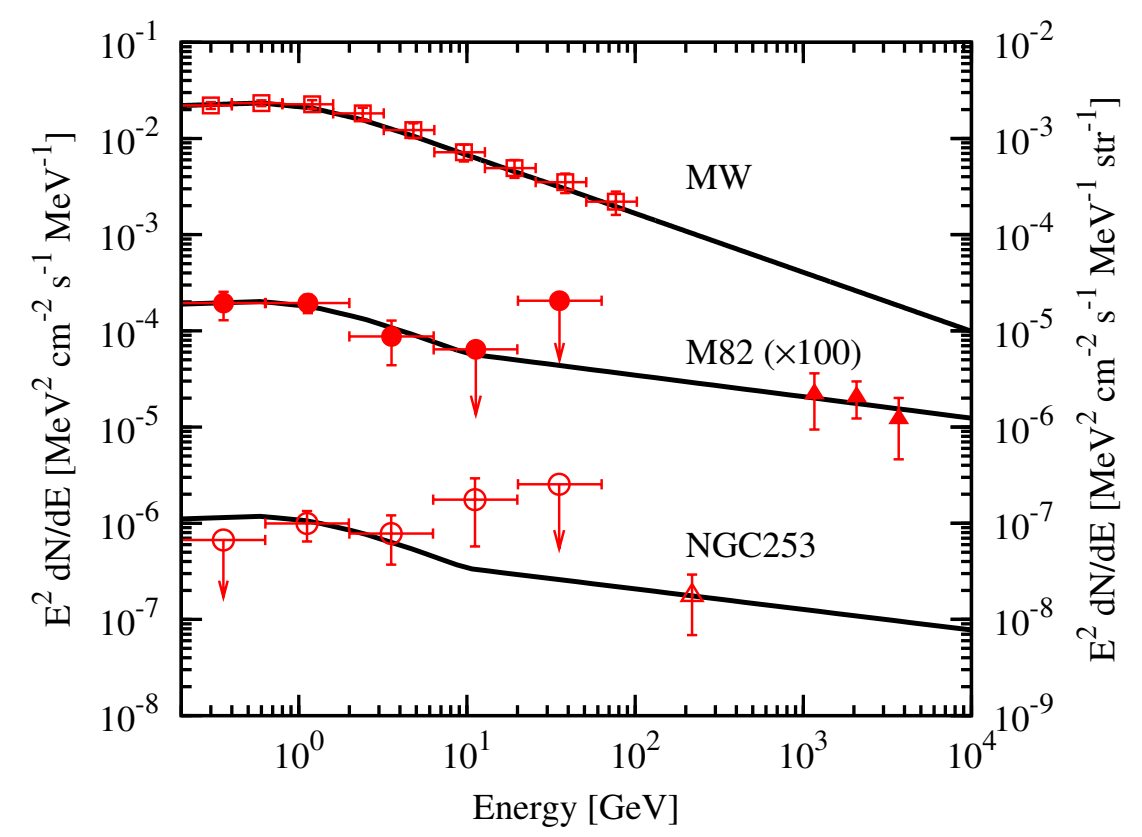

Figure 2: $\gamma$-ray spectrum of galaxies. MW is in units shown on the right ordinate, while the others are in left ordinate. 


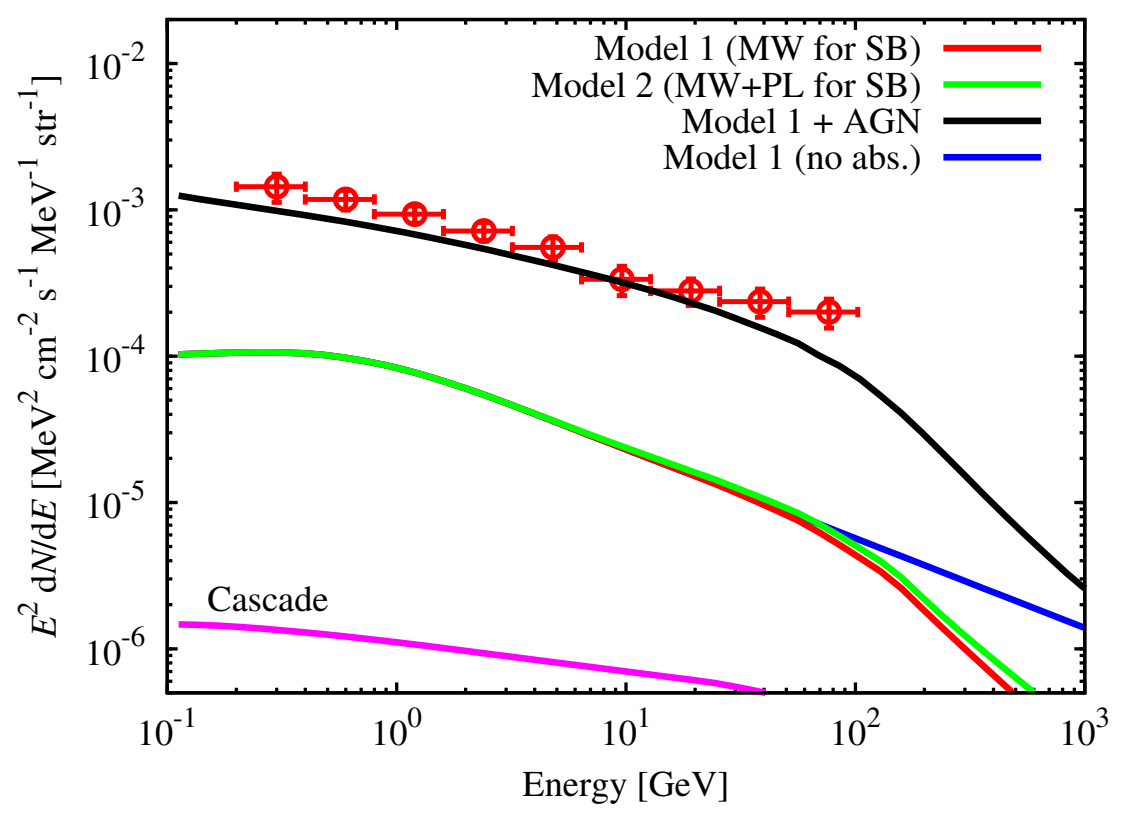

Figure 3: The EGRB spectrum.

plotted. As a result, the EGRB photon flux from all star-forming galaxies become $7 \%$ of the total EGRB, and the sum of the contributions from AGNs and star-forming galaxies account for the $50 \%$ of the total EGRB. It should also be noted that the combined spectrum by AGNs and star-forming galaxies is remarkably similar to the observed EGRB spectrum. Further examination is required to see whether the $50 \%$ residual of the EGRB is mainly a result of modeling uncertainty, experimental uncertainty, or significant contributions from other sources, e.g., dark matter annihilation.

\section{Summary}

We have presented a new calculation of EGRB from cosmic-ray interactions in star-forming galaxies, based on a state-of-the-art galaxy formation model in the framework of hierarchical structure formation. We found that star-forming galaxies make $\sim 7 \%$ contribution to the total EGRB flux reported by Fermi in our standard model. Combined with the contribution from blazars as estimated by the Fermi data, more than $50 \%$ of EGRB can be accounted for. The combined spectrum by AGNs and star-forming galaxies is remarkably similar to the observed EGRB spectrum.

More detailed description of our model and precise discussion of model uncertainties can be found in Makiya et al. (2011) [10].

\section{Acknowledgments}

This work was supported by the Grant-Aid for the Global COE Program "The Next Generation of Physics, Spun from University and Emergence " from the Ministry of Education, Culture, Sports, Science and Technology (MEXT) of Japan. The numerical calculations were in part carried 
out on SGI Altix3700 BX2 at Yukawa Institute for Theoretical Physics of Kyoto University. TT was supported by the Grant-in-Aid for Scientific Research (19047003, 19740099, 2004005) from MEXT. MARK was supported by the Research Fellowship for Young Scientists from the Japan Society for the Promotion of Science (JSPS).

\section{References}

[1] Fichtel, C. E., Hartman, R. C., Kniffen, D. A., Thompson, D. J., Ogelman, H. B., Ozel, M. E., \& Tumer, T. 1977, ApJ, 217, L9

[2] Fichtel, C. E., Simpson, G. A., \& Thompson, D. J. 1978, ApJ, 222, 833

[3] Acero, F., et al. 2009, Science, 326, 1080

[4] VERITAS Collaboration 2009, Nature, 462, 770

[5] Abdo, A. A., et al. 2010, ApJ, 709, L152

[6] Nagashima, M., \& Yoshii, Y. 2004, ApJ, 610, 23

[7] Nagashima, M., Yahagi, H., Enoki, M., Yoshii, Y., \& Gouda, N. 2005, ApJ, 634, 26

[8] Inoue, Y., \& Totani, T. 2009, ApJ, 702, 523

[9] Abdo, A. A., et al. 2010b, Phys. Rev. Lett., 104, 101101

[10] Makiya, R., Totani, T., \& Kobayashi, M. A. R. 2011, ApJ, 728, 158 